\title{
Genetic and bioinformatic analyses of the expression and function of PI3K regulatory subunit PIK3R3 in an Asian patient gastric cancer library
}

Jin Zhou', Geng Bo Chen ${ }^{3}$, Yew Chung Tang ${ }^{4}$, Rohit Anthony Sinha', Yonghui Wu ${ }^{8}$, Chui Sun Yap ${ }^{1}$, Guihua Wang ${ }^{5}$, Junbo Hu${ }^{5}$, Xianmin $\mathrm{Xia}^{5}$, Patrick Tan ${ }^{2,8,9,10}$, Liang Kee Goh ${ }^{3,6,7}$ and Paul Michael Yen ${ }^{1 *}$

\begin{abstract}
Background: While there is strong evidence for phosphatidylinositol 3-kinase (PI3K) involvement in cancer development, there is limited information about the role of PI3K regulatory subunits. PIK3R3, the gene that encodes the PI3K regulatory subunit p55y, is over-expressed in glioblastoma and ovarian cancers, but its expression in gastric cancer (GC) is not known. We thus used genetic and bioinformatic approaches to examine PIK3R3 expression and function in GC, the second leading cause of cancer mortality world-wide and highly prevalent among Asians.

Methods: Primary GC and matched non-neoplastic mucosa tissue specimens from a unique Asian patient gastric cancer library were comprehensively profiled with platforms that measured genome-wide mRNA expression, DNA copy number variation, and DNA methylation status. Function of PIK3R3 was predicted by IPA pathway analysis of co-regulated genes with PIK3R3, and further investigated by siRNA knockdown studies. Cell proliferation was estimated by crystal violet dye elution and BrdU incorporation assay. Cell cycle distribution was analysed by FACS.

Results: PIK3R3 was significantly up-regulated in GC specimens $(n=126, p<0.05)$, and 9.5 to $15 \%$ tumors showed more than 2 fold increase compare to the paired mucosa tissues. IPA pathway analysis showed that PIK3R3 promoted cellular growth and proliferation. Knockdown of PIK3R3 decreased the growth of GC cells, induced G0/G1 cell cycle arrest, decreased retinoblastoma protein (Rb) phosphorylation, cyclin D1, and PCNA expression.

Conclusion: Using a combination of genetic, bioinformatic, and molecular biological approaches, we showed that PIK3R3 was up-regulated in GC and promoted cell cycle progression and proliferation; and thus may be a potential new therapeutic target for GC.
\end{abstract}

\section{Background}

Class IA Phosphatidylinositol 3'-kinases (PI3K) is a heterodimer that consists of a p110 catalytic subunit and a p85 regulatory subunit. The catalytic subunit isoforms $\mathrm{p} 110 \alpha, \mathrm{p} 110 \beta$ and $\mathrm{p} 110 \delta$ are encoded by three genes PIK3CA, PIK3CB and PIK3CD, respectively [1]. The role of catalytic subunits in a wide range of cellular processes associated with cancer development and progression is well established [2]. The PIK3CA gene is one of the best

\footnotetext{
* Correspondence: paul.yen@duke-nus.edu.sg

'Laboratory of Hormonal Regulation, Cardiovascular and Metabolic Disorders Program, Duke-National University of Singapore Graduate Medical School, Singapore

Full list of author information is available at the end of the article
}

studied oncogenes, and is amplified, overexpressed, or frequently mutated in many cancers, including GC $[2,3]$. PIK3CB is the principal isoform involved in mediating PTEN-deficient tumourigenesis [4]. In addition, PIK3CD has also emerged as a key therapeutic target for haematological malignancies [5], notably acute myeloid leukaemia (AML). Consequently, targeting catalytic subunits represents an important strategy for the development of novel cancer therapeutics.

In contrast, current understanding of the role of regulatory subunits in tumorigenesis has been limited. Three genes, PIK3R1, PIK3R2 and PIK3R3, encode the p85 $\alpha$, $\mathrm{p} 85 \beta$ and $\mathrm{p} 55 \gamma$ isoforms of the $\mathrm{p} 85$ regulatory subunit, respectively [6]. PIK3R1, the inhibitory subunit of PI3K, 
is mutated in primary colorectal and endometrial cancer tumors $[7,8]$, and ectopic expression of some of those mutations increased the pAKT level in U2OS cells. Interestingly, PIK3R3 has increased expression in glioblastoma multiforme and ovarian cancer $[9,10]$. Knockdown of PIK3R3 inhibits IGF2-induced cell growth in glioblastoma multiforme [9] and induces apoptosis in ovarian cancer cells [10]. Taken together, these results suggest an oncogenic role for PIK3R3 in these cancers.

$\mathrm{GC}$ is the second leading cause of global cancer mortality and is highly prevalent among Asians [11]. Most GC patients are diagnosed with late stage disease and the overall 5-year survival rate is $<24 \%$ [12-14]. Deregulation of canonical oncogenic pathways such as E2F, K-RAS, p53, and Wnt/b-catenin signaling are known to occur with varying frequencies in GC [15-17], suggesting that GC is a heterogeneous disease with multiple molecular defects. Although PIK3R3 is overexpressed in several cancers, little is known about the expression and functional role of PIK3R3 in GC. To address these issues, we used genetic and bioinformatic approaches to interrogate a unique library of 126 paired GC samples and matched nonneoplastic mucosa tissues from Asian GC patients.

\section{Methods}

\section{Human cancer specimens and cell lines}

We created a library of 126 primary gastric tumors and their matched non-neoplastic mucosa tissues from 126 Asian patients that were originally stored at the SingHealth Tissue Repository, an institutional resource of National Cancer Centre of Singapore and Singapore General Hospital. All patient samples were obtained with informed patient consent and approvals from Institutional Review Boards and Ethics Committees. GC cell lines HGC-27, KATO III, AGS cells were purchased from the American Type Culture Collection. MKN7, TMK1 and IM95 cells were obtained from the Japan Health Science Research Resource Bank. Cell lines were maintained in a humidified atmosphere containing $5 \% \mathrm{CO} 2$ at $37^{\circ} \mathrm{C}$. HGC-27, IM95 cells were cultured in DMEM medium supplemented with $10 \%$ FBS (Sigma); AGS, TMK1 and KATO III and MKN7 were cultured in RPMI 1640 medium supplemented with $10 \%$ FBS.

\section{Microarray Profiling \& Pre-processing}

Genomic DNA was extracted from flash-frozen tissues using a Qiagen genomic DNA extraction kit. Total RNAs was extracted using Trizol (Invitrogen, CA), digested with RNase free DNase (RQ1 DNase, Promega), and subsequently purified using an RNeasy Mini kit (Qiagen, CA). Genome-wide mRNA expression was profiled using Affymetrix GeneChip Human Genome U133 Plus 2.0 array. Copy number analysis was profiled using
Affymetrix Human SNP array 6.0. DNA methylation analysis was profiled using Illumina Infinium methylation assay. Profiling on the individual microarray platforms was done according to manufacturer's specifications.

The raw microarray data was pre-processed using the respective platform software from the manufacturer: mRNA and SNP data using Affymetrix's Genome Studio and Genotyping Console respectively, and DNA methylation using Illumina's BeadStudio. For SNP data, the normal gastric samples were used as the reference for normalization. The copy number segmentation was generated using Circular Binary Segmentation (CBS) from the $\mathrm{R}$ package DNA copy [18] using default settings. Gene-based copy number alterations were obtained by averaging the segments within each gene. Amplification and deletion were called using the threshold of 0.3 and -0.3 respectively [19].

The microarray data has been deposited into the National Centre for Biotechnology Information's (NCBI) Gene Expression Omnibus (GEO) website, series accession number GSE31168 (SNP6) and GSE15460 (mRNA).

\section{PIK3R3 co-regulated gene and IPA pathway analysis}

Co-regulated genes with PIK3R3 were elucidated using Pearson correlation on the mRNA data. We focused on probes that were specifically targeting the genes (i.e., probes with suffix '_at' and '_a_at') and significant correlation after correcting for multiple testing $(\mathrm{p}<1 \mathrm{e}-5)$. Pathways involving the probes were assessed using Ingenuity Pathway Analysis software (http://www.ingenuity. com). All statistical analyses were computed using the $R$ statistical package.

\section{RNA Extraction and RT-PCR}

Total cellular RNA was isolated with the High Pure RNA isolation Kit (Roche) following the manufacturer's protocol and total RNA was quantified with a Nanodrop ND-1000 spectrophotometer. Total RNA $(1 \mu \mathrm{g})$ was reverse-transcribed using iSCRIPT cDNA synthesis kit (Bio-rad) under condtions defined by the supplier. cDNA was quantified by real-time PCR on the Rotor-Gene Q System (Qiagen). PIK3R3 forward primer: GAGAGGG GAATGAAAAGGAGA, and reverse primer: ATCAT GAATCTCACCCAGACG [10]. $\beta$-actin forward primer: AGAGCCTCGCCTTTGCCGAT, and reverse primer: TT GCACATGCCGGAGCCGTT; GAPDH forward primer: TCTTTTGCGTCGCCAGCCGA, and reverse primer: CCAGGCGCCCAATACGACCA. PCR was done using QuantiFast SYBR Green PCR Kit (Qiagen) according to manufacturer's instructions.

To test the effect of LY294002 on PIK3R3 expression, the complete medium was replaced by serum free medium and LY294002 (20 $\mu \mathrm{M} / \mathrm{L}$, Sigma $)$ was added. The total RNA was extracted $24 \mathrm{~h}$ after LY294002 treatment. 


\section{PIK3R3 siRNA transfection}

Two PIK3R3 siRNAs ordered from Santa Cruz (sc-39124) or Invitrogen (s16152) were used to knock down PIK3R3 in GC cells. HGC-27 or TMK1 cells were seeded into 12 or 6-well plates in complete medium and cultured overnight. Then the medium was replaced with opti-MEM medium (Invitrogen) containing PIK3R3 or control siRNA and Lipofectamine RNAimax (Invitrogen) according to the manufacturer's recommendations. $48 \mathrm{~h}$ after transfection, cell lysates were prepared for Western blot analysis for detection of PIK3R3 expression.

\section{Cell proliferation assay}

Cell proliferation assay was done as previously described $[20,21]$. Briefly, $24 \mathrm{~h}$ after siRNA transfection, the HGC27 cells were trypsinized, resuspended in $1.1 \mathrm{ml}$ complete culture medium, and re-seeded into 4 new 12 -well plates at $250 \mu \mathrm{l}$ per well. The cells were fixed 1 to 4 days after reseeding, respectively, and stained with crystal violet. Using $10 \%$ acetic acid, dye was extracted, and absorbance at $595 \mathrm{~nm}$ was measured using a multiwall spectrophotometer (Bio-Rad).

\section{Measurement of BrdU incorporation}

$48 \mathrm{~h}$ after siRNA transfection, HGC27 cells cultured in 6 well-plates were incubated with $\operatorname{BrdU}(10 \mu \mathrm{g} / \mathrm{ml})$ for $7 \mathrm{~min}$. The cells were detached, fixed and stained using FITC BrdU Flow Kit (BD Pharmingen) following the manufacturer's protocol. DNA synthesis was determined by flow cytometer.

\section{Annexin-V/propidium iodide staining}

Annexin V staining was detected by flow cytometry using the FITC Annexin V Apoptosis Detection Kit I (BD Pharmingen). $48 \mathrm{~h}$ after siRNA transfection, Both floating and adherent HGC27 cells were collected. Then the cells were washed and stained following the manufacturer's protocol. Apoptotic cells were determined by flow cytometer.

\section{Cell cycle analysis}

$48 \mathrm{~h}$ after siRNA transfection, HGC-27 cells were harvested, washed twice with cold phosphate buffered saline (PBS, pH 7.4), and fixed with $70 \%$ ethanol $/ 30 \% \mathrm{PBS}$ at $4^{\circ} \mathrm{C}$ overnight. The fixed cells were incubated with $0.5 \mathrm{ml}$ PBS containing $10 \mu \mathrm{g} / \mathrm{ml} \mathrm{RNase}$ for $30 \mathrm{~min}$ at $37^{\circ} \mathrm{C}$, then stained with $20 \mu \mathrm{g} / \mathrm{ml}$ propidium iodide (PI, Sigma) for $30 \mathrm{~min}$ in the dark at room temperature, and finally analyzed on a FACS cytometer (Beckman FC5000). A minimum of $1 \times 10^{4}$ cells/ sample was evaluated.

\section{Western blot analysis}

Cells were harvested, and lysed $48 \mathrm{~h}$ after siRNA transfection. Proteins were separated by SDS-PAGE under reducing conditions and transferred to nitrocellulose membranes. Membranes were blocked with $5 \%$ nonfat milk in phosphate-buffered saline with $0.1 \%$ tween 20 (PBST), and the membranes for PI3KR3 blot were blocked with $2 \%$ gelatine, $0.2 \%$ FBS in PBST [22]. The blots were incubated overnight at $4^{\circ} \mathrm{C}$ with the following antibodies: anti-PIK3R3 antibody, anti-beta actin antibody (Santa Cruz), anti-phospho-RB (Ser 780), anti-cyclin D1, antiphospho-p38 MAPK antibody (Thr180/Tyr 182), antiphospho-AKT (Ser 473), anti-phospho-AKT (Ser 308), anti-phospho-m-TOR (Ser 2448) (Cell signaling Technology), anti-PCNA antibody (Millipore). Immunoblot analysis was performed using an enhanced chemiluminescence procedure (GE Healthcare).

\section{Results}

\section{PIK3R3 mRNA is over-expressed in a subgroup of GC}

We examined the PIK3R3 mRNA expression of 126 gastric adenocarcinoma samples and matched non-neoplastic mucosa control gastric tissues, profiled on Affymetrix GeneChip Human Genome U133 Plus 2.0 arrays. We identified cancers that expressed elevated PIK3R3 by comparing PIK3R3 mRNA expression in the tumor samples with their expression in control tissues from the same patients. Two probe sets (202743_at and 211580_s_at) interrogated PIK3R3 mRNA levels in these arrays, and thus provided two independent read-outs of PIK3R3 gene expression. PIK3R3 was significantly up-regulated in cancer specimens $(\mathrm{p}<0.05)$ and considered over-expressed if the probe sets measured a greater than 2-fold increase in PIK3R3 expression in the tumor sample compared to its control. Thus, $19(15 \%)$ or $12(9.5 \%)$ samples exhibited PIK3R3 over-expression according to different probe sets (Figure 1).

\section{Knockdown of PIK3R3 expression decreases cell proliferation in GC cells}

In order to understand the potential role of PIK3R3 in GC, pathway analyses (Ingenuity IPA software) was performed on genes co-regulated with PIK3R3. Pathway analysis results highly suggested that PIK3R3 was involved in cell growth and proliferation (Additional file 1: Figure S1). To further investigate the potential effect of PIK3R3 expression on cell proliferation, we first tested the expression of PIK3R3 in $6 \mathrm{GC}$ cell lines (Figure 2A). HGC27, TMK1 and IM95 had significantly higher PIK3R3 expression than KATO III, AGS and MNK7. We next used PIK3R3 siRNA to specifically knock down PIK3R3 protein expression in HGC27 GC cells, and confirmed the knockdown efficiency by Western blotting (Figure 2B). Knockdown of PIK3R3 significantly decreased cell proliferation based upon both crystal violet dye elution and BrdU incorporation assay (Figures $2 \mathrm{C}$ and $\mathrm{D}$ ). Additionally, PIK3R3 knockdown did not increase in the early apoptotic 


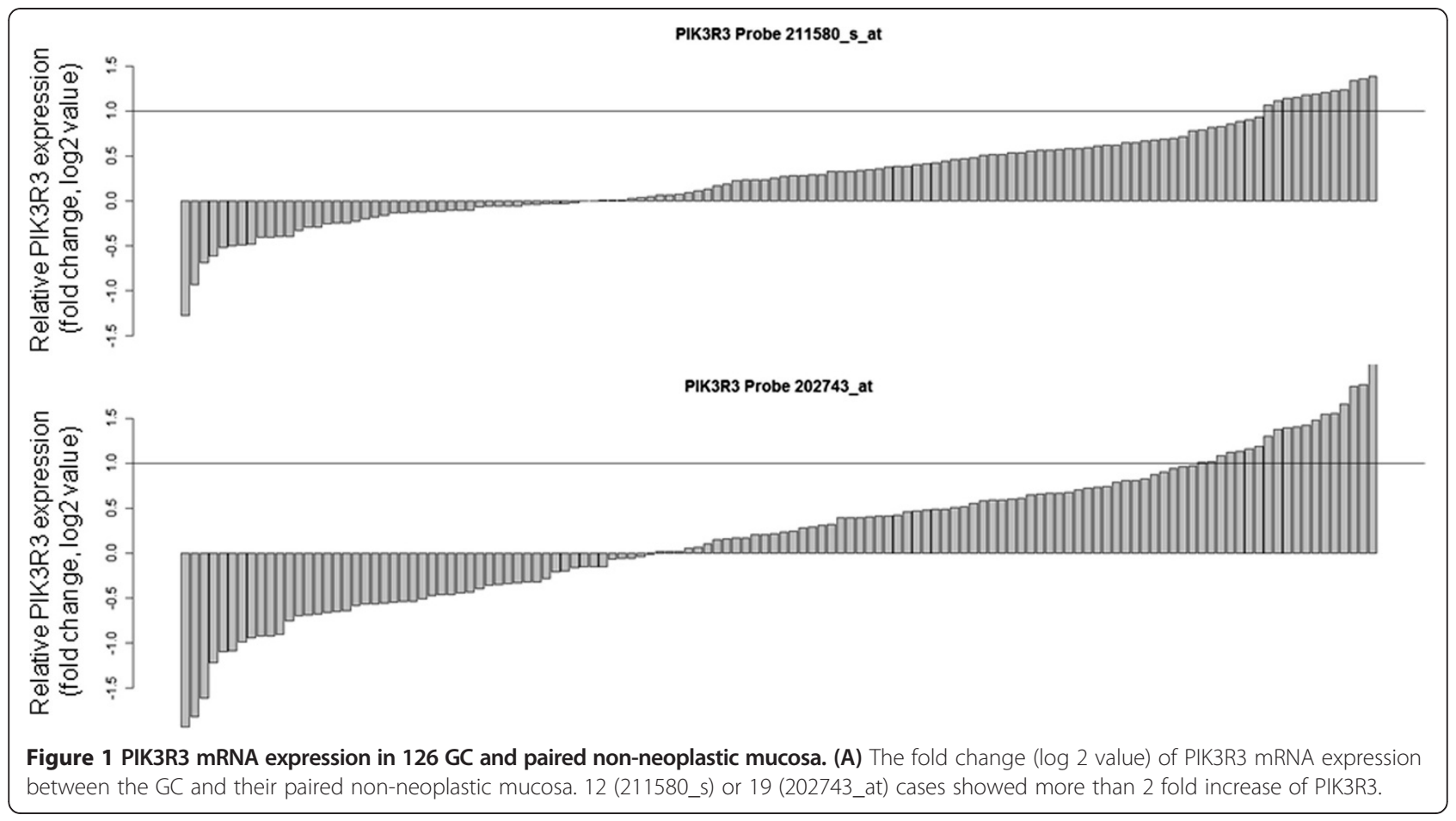

cell populations (Figure 2E). The results were confirmed in HGC27 cells using another PIK3R3 siRNA (Additional file 2: Figure S2).

\section{Knockdown of PIK3R3 causes G0/G1 cell cycle arrest}

Since PIK3R3 knockdown decreased cell proliferation, we examined whether PIK3R3 knockdown also caused cell cycle arrest at the G0/G1 phase by PI staining and FACS analyses (Figure 3A). We observed that PIK3R3 knockdown increased cells in $G_{0} / G_{1}$ arrest (59.8\% vs. $\left.48.4 \%\right)$. Furthermore, the percentage of knockdown cells in $\mathrm{G}_{0} / \mathrm{G}_{1}$ cell cycle arrest increased when cells were deprived of growth factors in culture media conditions (Figure 3A). We also found increased G0/G1 cell cycle arrest in TMK1 GC cells (Additional file 3: Figure S3A). Taken together, our results suggest that the main effect of PIK3R3 knockdown is inhibition of cell proliferation by G0/G1 cell cycle arrest rather than induction of cell apoptosis.

\section{Knockdown of PIK3R3 decreases Rb phosphorylation and cyclin D1 protein expression but does not affect basal pAkt level}

To understand better the functional role of PIK3R3 on cell proliferation, we examined the expression of various molecular markers in the PIK3R3 knockdown cells. We found reduced cyclin D1 levels and decreased phosphorylation of $\mathrm{Rb}$ protein, both of which are associated with G0/G1 arrest (Figure 3B and Additional file 3: Figure S3B). Expression of the cell proliferation marker, PCNA, also was reduced (Figure 3B). Interestingly, phosphorylation of Akt and mTOR was unaffected by PIK3R3 knockdown (Figure 3B and Additional file 3: Figure S3), suggesting that overexpression of PIK3R3 does not primarily drive cell proliferation through PI3K-pAkt signaling.

\section{PIK3R3 over-expression can be regulated by PIK3CA}

47 of the 126 paired GC and control tissues were comprehensively profiled on multi-omics platforms that measured genome-wide mRNA expression, DNA copy number variation, and DNA methylation status. This multi-platform allowed us to investigate whether structural genomic changes or epigenetic regulation accounted for the upregulation of PIK3R3. However, we did not observe any PIK3R3 gene amplification (i.e. average segments within the gene were $<0.3$, Additional file 4: Figure $\mathrm{S} 4 \mathrm{~A}$ ), or $\mathrm{CPG}$ island de-methylation in the GC with high PIK3R3 mRNA expression (Additional file 4: Figure S4B). Additionally, there was no consistent significant correlation between either PIK3R3 copy number and mRNA level, or CpG island methylation and the mRNA expression level (Additional file 4: Figure S4). Taken together, these results indicate that some other mechanism accounted for the increased PIK3R3 mRNA expression in the high-expressing GC. Recently, several studies have suggested that activated PI3K signaling induces the expression of PIK3R3 [23,24]. We thus investigated this possibility by assessing the correlation of mRNA expression of PIK3R3 and PIK3CA, the p110 $\alpha$ catalytic subunit of PI3K. 


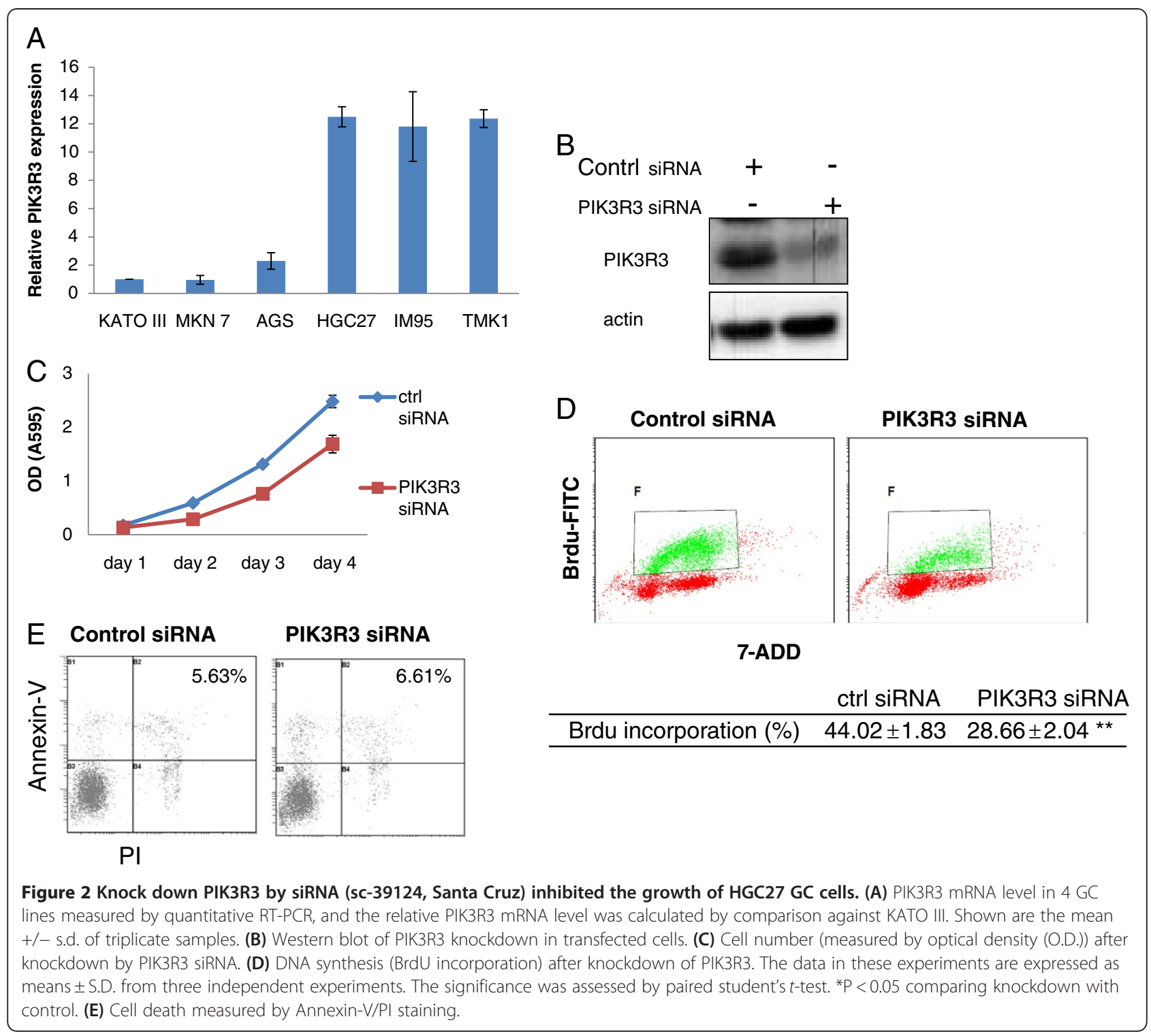

We found that PIK3CA and PIK3R3 showed a significant positive correlation in 126 GC (Additional file 5: Figure S5). Furthermore, PIK3CA inhibitor LY294002 treatment decreased PIK3R3 mRNA and protein levels in HGC27 and IM95 cells (Figure 4).

\section{Discussion}

Although the roles of the PI3K catalytic subunit in tumorigenesis is well established, current understanding of the function of PI3K regulatory subunits such as PIK3R3 is still limited. A previous study showed that PIK3R3 gene had copy number gain in high-grade glioma, and the Comparative Genomic Hybridization $(\mathrm{CGH})$ ratios for this locus were positively-correlated with proliferation signatures [25]. Furthermore, the IGF2-PIK3R3 signaling axis was involved in promoting the growth of a subclass of highly aggressive human glioblastomas [9]. In this study, we compared PIK3R3 expression in 126 paired GC with non-neoplastic gastric mucosa control tissues from the same patients, and found that approximately $9.5 \%$ to $15 \%$ of GC showed increased PIK3R3 expression. IPA pathway analysis suggested a key role of PIK3R3 in cell proliferation. Knockdown of PIK3R3 by siRNA decreased HGC27 GC cell proliferation and induced cell cycle blockade at $G_{0} / G_{1}$. Taken together, these results suggest that PIK3R3 stimulates cell proliferation in GC and may be a potential therapeutic target for a subgroup of GC.

PIK3R3 shares significant sequence identity with PIK3R1 and PIK3R2 regulatory subunit in a proline-rich motif and two Src homology 2 (SH2) domains; however, it has an unique 24-residue NH2 terminus [26]. PIK3R3 


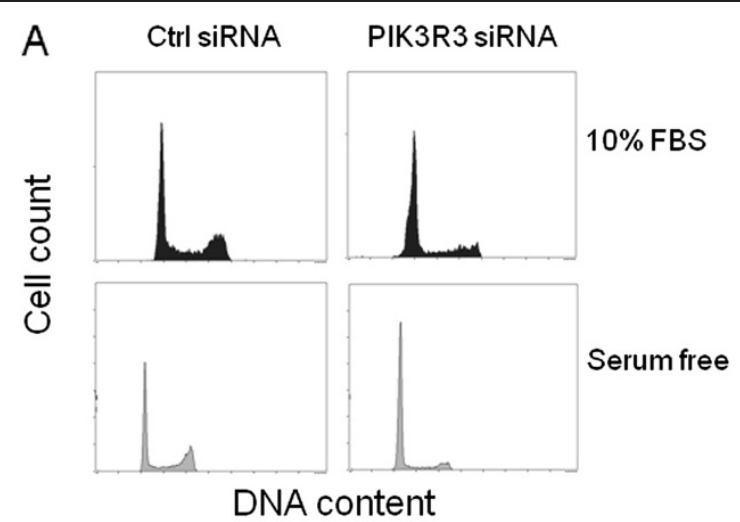

B

\begin{tabular}{|c|c|c|c|}
\hline & & Ctrl siRNA & PIK3R3 siRNA \\
\hline \multirow{3}{*}{$10 \%$ FBS } & G0/G1 & $48.40 \pm 2.38$ & $59.76 \pm 2.76^{* *}$ \\
\cline { 2 - 4 } & $\mathrm{S}$ & $23.09 \pm 0.52$ & $18.45 \pm 1.76^{*}$ \\
\cline { 2 - 4 } & $\mathrm{G} 2 / \mathrm{M}$ & $24.74 \pm 2.71$ & $15.37 \pm 2.24^{* *}$ \\
\hline \multirow{3}{*}{ Serumfree } & $\mathrm{G} 0 / \mathrm{G} 1$ & $49.48 \pm 2.45$ & $74.25 \pm 1.48^{* *}$ \\
\cline { 2 - 4 } & $\mathrm{S}$ & $17.82 \pm 4.30$ & $10.02 \pm 1.45^{*}$ \\
\cline { 2 - 4 } & $\mathrm{G} 2 / \mathrm{M}$ & $29.79 \pm 5.36$ & $13.08 \pm 1.90^{*}$ \\
\hline
\end{tabular}

\section{Ctrl SiRNA}

PIK3R3 SIRNA

p-RB (ser 780)

Cyclin D1

PCNA

p-P38 (Thr180/Tyr182)

p-AKT (ser 473)

p-AKT (ser 308)

p-mTOR (ser2448)

PIK3R3

actin
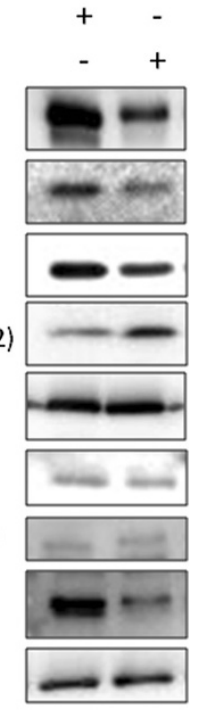

Figure 3 Effects of PIK3R3 knockdown on cell cycle progression and cell signaling of HGC27 GC cells. (A) The effect of PIK3R3 knockdown on cell cycle progression in HGC27 cells. The cells were cultured in complete medium for $48 \mathrm{~h}$ or $24 \mathrm{~h}$ followed by another $24 \mathrm{~h}$ in serum free medium after siRNA transfection. The cells then were harvested for cell cycle analysis. The data are expressed as means \pm S.D. from three independent experiments. The significance was assessed by paired student's $t$-test. ${ }^{*} \mathrm{P}<0.05$ and ${ }^{* *} \mathrm{P}<0.01$ compared with control. (B) Western blot analysis of the effects of PIK3R3 knockdown on cell signaling. Total cell extracts were probed with antibodies to cyclin D1, phospho-Rb (p-Rb), PCNA, phospho-AKT (p-AKT), phospho-mTOR (p-mTOR), PIK3R3, actin (loading control) as indicated.

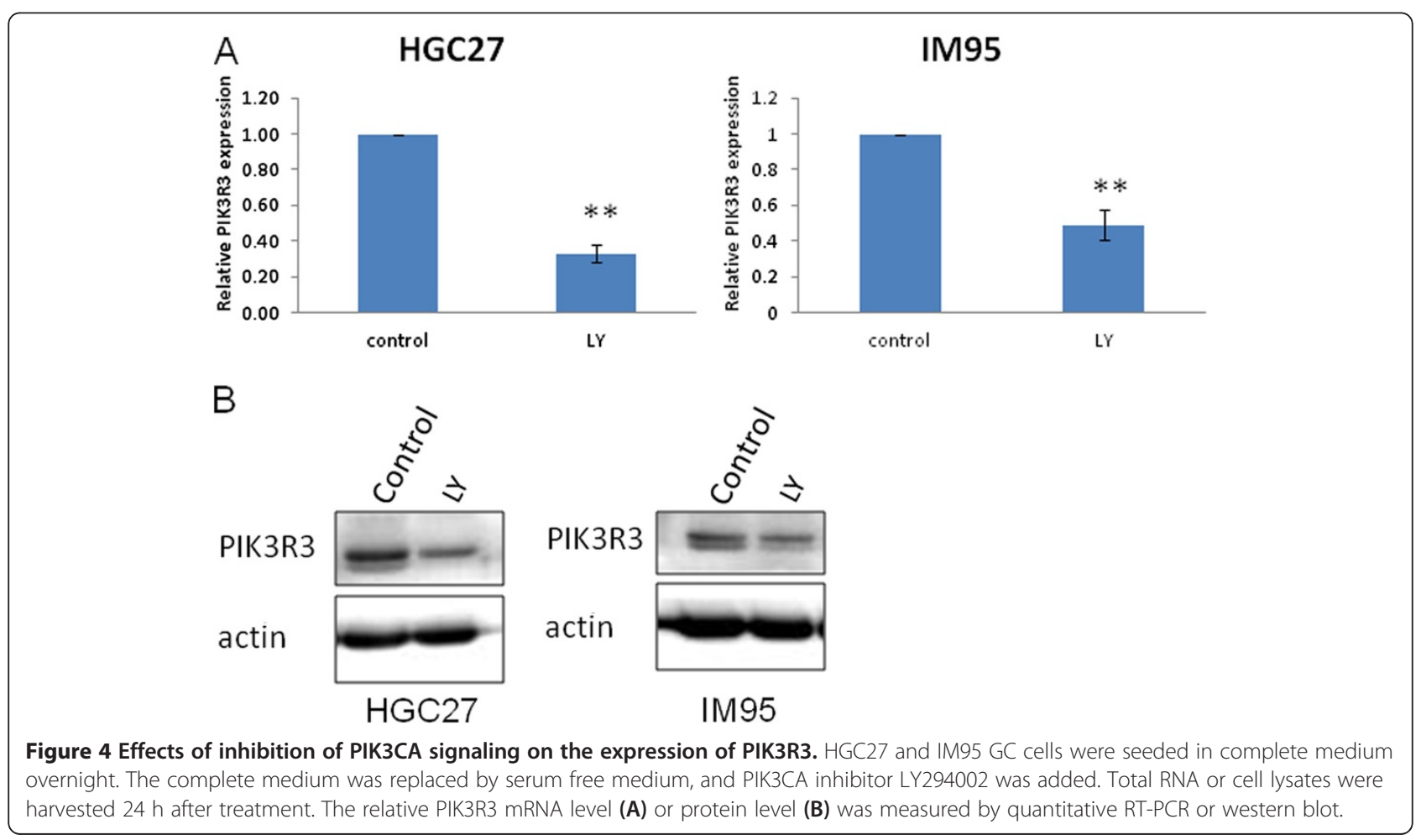


binds to $\mathrm{Rb}$ via this unique region as the $\mathrm{N}$-terminal 24 amino acids of PIK3R3 (N24) are sufficient for binding to $\mathrm{Rb}[27,28]$. Ectopic expression of N24 also inhibited cyclin D1 and $E$ promoter activity and caused $G_{0} / G_{1}$ phase arrest in several cancer cell lines [27]. Likewise, in the current study, knockdown of PIK3R3 caused $G_{0} / G_{1}$ cell cycle arrest in HGC27 and TMK1 GC cells. Consistent with this observation, Rb phosphorylation, cyclin D1, and PCNA protein levels also were decreased after PIK3R3 knockdown. Interestingly, the pAkt levels did not change after PIK3R3 knockdown. Additionally, when PIK3R3 was overexpressed in AGS cells, a low PIK3R3expressing gastric cancer cell line, we did not observe any significant change in phosphorylation of Akt (data not shown). Taken together, these findings show that both increased and decreased expression of PIK3R3 do not affect pAkt levels in these cell lines under our experimental conditions. Of note, we have observed similar effects in several colon cancer cell lines and MCF7 breast cancer cells ([29]and unpublished data). This lack of change in pAkt levels may be due to either preferential nuclear localization of PIK3R3 [27] or low PIK3R3 expression relative to $\mathrm{p} 85$ regulatory subunit isoforms. In contrast to our findings, Soroceau et al. found decreased pAkt in G96 glioblastoma cells after PIK3R3 knockdown which may be due to different experimental conditions or different tissue origins of cell lines as PIK3R3 expression is high in the brain [9]. Nevertheless, our current and previous studies suggest that PIK3R3 can promote cell growth through a novel signaling pathway by regulating $\mathrm{Rb}$ phosphorylation and cyclin D1.

Finally, we investigated the mechanism of PIK3R3 over-expression in GC. Zhang et al. found PIK3R3 was over-expressed in ovarian cancer due to gene copy number gain [10]. However, no gene amplification or DNA de-methylation of PIK3R3 gene was found in our set of GC samples. Interestingly, Demoulin et al. found that PDGF activated transcription factor SREBP-1 in a PI3Kdependent manner [23], and ectopic expression of SREBP-1 induces the expression of PIK3R3 in AG01518 human foreskin fibroblasts [24]. Therefore, these results suggested that activated PI3K signaling could induce the expression of PIK3R3. Indeed, we observed a positive correlation between PIK3CA and PIK3R3 mRNA expression in our $126 \mathrm{GC}$ samples. Furthermore, the PIK3CA inhibitor, LY294002 decreased PIK3R3 expression in HGC27 and IM95 GC cell lines. Our results thus provide direct experimental evidence that PIK3R3 expression can be regulated by PIK3CA.

\section{Conclusion}

The in silico and molecular biological approaches used in the present study have provided a better understanding of the expression and role of PIK3R3 in GC. The identification of PIK3R3 as a proliferation-promoting factor in GC suggests that it might be a potential therapeutic target for a subset of GC.

\section{Additional files}

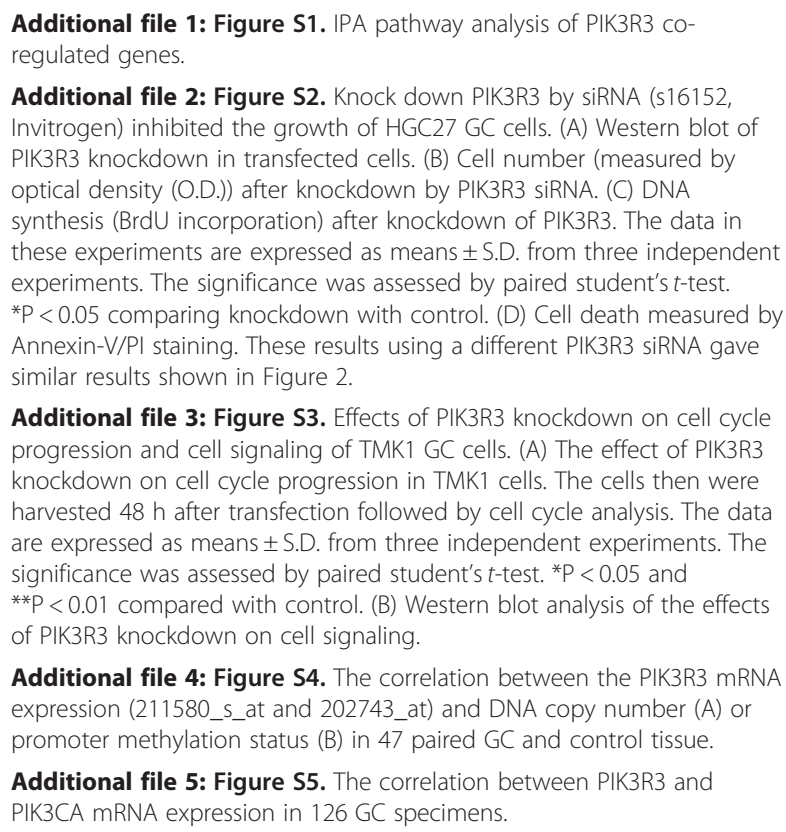

Additional file 3: Figure S3. Effects of PIK3R3 knockdown on cell cycle progression and cell signaling of TMK1 GC cells. (A) The effect of PIK3R3 knockdown on cell cycle progression in TMK1 cells. The cells then were harvested $48 \mathrm{~h}$ after transfection followed by cell cycle analysis. The data are expressed as means \pm S.D. from three independent experiments. The significance was assessed by paired student's $t$-test. * $P<0.05$ and ${ }^{*} \mathrm{P}<0.01$ compared with control. (B) Western blot analysis of the effects of PIK3R3 knockdown on cell signaling.

Additional file 4: Figure S4. The correlation between the PIK3R3 mRNA expression (211580_s_at and 202743_at) and DNA copy number (A) or promoter methylation status (B) in 47 paired GC and control tissue.

Additional file 5: Figure S5. The correlation between PIK3R3 and PIK3CA mRNA expression in 126 GC specimens.

\section{Abbreviations}

PI3K: phosphatidylinositol 3-kinase; AML: acute myeloid leukaemia; GC: Gastric cancer; CBS: Circular Binary Segmentation; PI: propidium iodide; PBST: phosphate-buffered saline with 0.1\% Tween 20; CGH: Comparative Genomic Hybridization; SH2: Src homology 2; N24: N-terminal 24 amino acid of PIK3R3.

\section{Competing interests}

The authors declare that they have no competing interests.

\section{Authors' contributions}

JZ contributed to the lab work, interpreted the results and wrote the paper. GBC, YCT, and YW did statistical analyses of the data. RAS, CSY, GW, JH, and $X X$ assisted the laboratory work. PT did the data collection. LKG designed the data analysis, interpreted the results and wrote the paper. PMY interpreted the results and wrote the paper. All authors have read and approved the final manuscript.

\section{Acknowledgements}

The authors would like to thank Mr. Sherwin Ying Xie, Dr. Mobin M. Siddique (Cardiovascular and Metabolic Disorders Program, Duke-NUS Graduate Medical School) for their helpful advice and constructive criticisms. This work was supported by NMRC and Duke-NUS Graduate Medical School Faculty Funds sponsored by the Ministry of Health, Ministry of Education, and Ministry of Trade, Singapore and A*StaR to PMY and PT, and Khoo Discovery Award (KDP/2008/0002 and KDP/2009/0006) to LKG.

\section{Author details}

${ }^{1}$ Laboratory of Hormonal Regulation, Cardiovascular and Metabolic Disorders Program, Duke-National University of Singapore Graduate Medical School, Singapore. ${ }^{2}$ Laboratory of Genomic Oncology, Cancer and Stem Cell Program, Duke-National University of Singapore Graduate Medical School, Singapore. ${ }^{3}$ Laboratory of Computational Biology, Cancer and Stem Cell Program, Duke-National University of Singapore Graduate Medical School, Singapore. ${ }^{4}$ Laboratory of Computational Systems Biology and Human Genetics, Neuroscience and Behavioral Disorder Program, Duke-National 
University of Singapore Graduate Medical School, Singapore. ${ }^{5}$ Cancer Research Institute, Tongji Hospital, Huazhong University of Science and Technology, Wuhan 430030, China. ${ }^{6}$ Department of Medical Oncology, National Cancer Centre, Singapore. ' $5 a w$ Swee Hock School of Public Health, National University of Singapore, Singapore. ${ }^{8} \mathrm{Cellular}$ and Molecular Research, National Cancer Centre, Singapore. ${ }^{9}$ Cancer Science Institute of Singapore, National University of Singapore, Singapore. ${ }^{10} \mathrm{Genome}$ Institute of Singapore, Singapore.

Received: 26 November 2011 Accepted: 9 July 2012

Published: 9 August 2012

\section{References}

1. Fruman DA, Meyers RE, Cantley LC: Phosphoinositide kinases. Annu Rev Biochem 1998, 67:481-507.

2. Bader AG, Kang S, Zhao L, Vogt PK: Oncogenic PI3K deregulates transcription and translation. Nat Rev Cancer 2005, 5(12):921-929.

3. Samuels Y, Wang Z, Bardelli A, Silliman N, Ptak J, Szabo S, Yan H, Gazdar A, Powell SM, Riggins GJ, et al: High frequency of mutations of the PIK3CA gene in human cancers. Science 2004, 304(5670):554.

4. Wee S, Wiederschain D, Maira SM, Loo A, Miller C, deBeaumont R, Stegmeier F, Yao YM, Lengauer C: PTEN-deficient cancers depend on PIK3CB. Proc Natl Acad Sci USA 2008, 105(35):13057-13062.

5. Billottet C, Grandage VL, Gale RE, Quattropani A, Rommel C, Vanhaesebroeck $B$, Khwaja A: A selective inhibitor of the p110delta isoform of PI 3-kinase inhibits AML cell proliferation and survival and increases the cytotoxic effects of VP16. Oncogene 2006, 25(50):6648-6659.

6. Engelman JA, Luo J, Cantley LC: The evolution of phosphatidylinositol 3kinases as regulators of growth and metabolism. Nat Rev Genet 2006, 7(8):606-619.

7. Jaiswal BS, Janakiraman V, Kljavin NM, Chaudhuri S, Stern HM, Wang W, Kan $Z$, Dbouk HA, Peters BA, Waring P, et al: Somatic mutations in p85alpha promote tumorigenesis through class IA PI3K activation. Cancer Cell 2009, 16(6):463-474.

8. Urick ME, Rudd ML, Godwin AK, Sgroi D, Merino M, Bell DW: PIK3R1 (p85alpha) is somatically mutated at high frequency in primary endometrial cancer. Cancer Res 2011, 71(12):4061-4067.

9. Soroceanu L, Kharbanda S, Chen R, Soriano RH, Aldape K, Misra A, Zha J, Forrest WF, Nigro JM, Modrusan Z, et al: Identification of IGF2 signaling through phosphoinositide-3-kinase regulatory subunit 3 as a growthpromoting axis in glioblastoma. Proc Natl Acad Sci USA 2007, 104(9):3466-3471.

10. Zhang L, Huang J, Yang N, Greshock J, Liang S, Hasegawa K, Giannakakis A, Poulos N, O'Brien-Jenkins A, Katsaros D, et al: Integrative genomic analysis of phosphatidylinositol 3'-kinase family identifies PIK3R3 as a potential therapeutic target in epithelial ovarian cancer. Clin Cancer Res 2007, 13(18 Pt 1):5314-5321.

11. Devesa SS, Bray F, Vizcaino AP, Parkin DM: International lung cancer trends by histologic type: male:female differences diminishing and adenocarcinoma rates rising. Int J Cancer 2005, 117(2):294-299.

12. Sun W, Haller DG: Recent advances in the treatment of gastric cancer. Drugs 2001, 61(11):1545-1551.

13. Yoo CH, Noh SH, Shin DW, Choi SH, Min JS: Recurrence following curative resection for gastric carcinoma. Br J Surg 2000, 87(2):236-242.

14. Jemal A, Siegel R, Ward E, Murray T, Xu J, Thun MJ: Cancer statistics, 2007. CA Cancer J Clin 2007, 57(1):43-66.

15. Suzuki T, Yasui W, Yokozaki H, Naka K, Ishikawa T, Tahara E: Expression of the E2F family in human gastrointestinal carcinomas. Int J Cancer 1999, 81(4):535-538.

16. Hiyama T, Haruma K, Kitadai Y, Masuda H, Miyamoto M, Tanaka S, Yoshihara M, Shimamoto F, Chayama K: K-ras mutation in helicobacter pyloriassociated chronic gastritis in patients with and without gastric cancer. Int J Cancer 2002, 97(5):562-566.

17. Chen XY, Wang ZC, Li H, Cheng XX, Sun Y, Wang XW, Wu ML, Liu J: Nuclear translocations of beta-catenin and TCF 4 in gastric cancers correlate with lymph node metastasis but probably not with CD44 expression. Hum Pathol 2005, 36(12):1294-1301.

18. Olshen $A B$, Venkatraman ES, Lucito R, Wigler M: Circular binary segmentation for the analysis of array-based DNA copy number data. Biostatistics 2004, 5(4):557-572.
19. Beroukhim R, Getz G, Nghiemphu L, Barretina J, Hsueh T, Linhart D, Vivanco I, Lee JC, Huang JH, Alexander S, et al: Assessing the significance of chromosomal aberrations in cancer: methodology and application to glioma. Proc Natl Acad Sci USA 2007, 104(50):20007-20012.

20. Gayer CP, Chaturvedi LS, Wang S, Craig DH, Flanigan T, Basson MD: Straininduced proliferation requires the phosphatidylinositol 3-kinase/AKT/ glycogen synthase kinase pathway. J Biol Chem 2009, 284(4):2001-2011.

21. Zhou J, Chen Y, Lang JY, Lu JJ, Ding J: Salvicine inactivates beta 1 integrin and inhibits adhesion of MDA-MB-435 cells to fibronectin via reactive oxygen species signaling. Mol Cancer Res 2008, 6(2):194-204.

22. Kivi N, Greco D, Auvinen P, Auvinen E: Genes involved in cell adhesion, cell motility and mitogenic signaling are altered due to HPV 16 E5 protein expression. Oncogene 2008, 27(18):2532-2541.

23. Demoulin JB, Ericsson J, Kallin A, Rorsman C, Ronnstrand L, Heldin CH: Platelet-derived growth factor stimulates membrane lipid synthesis through activation of phosphatidylinositol 3-kinase and sterol regulatory element-binding proteins. J Biol Chem 2004, 279(34):35392-35402.

24. Kallin A, Johannessen LE, Cani PD, Marbehant CY, Essaghir A, Foufelle F, Ferre $\mathrm{P}$, Heldin CH, Delzenne NM, Demoulin JB: SREBP-1 regulates the expression of heme oxygenase 1 and the phosphatidylinositol-3 kinase regulatory subunit p55 gamma. J Lipid Res 2007, 48(7):1628-1636.

25. Phillips HS, Kharbanda S, Chen R, Forrest WF, Soriano RH, Wu TD, Misra A, Nigro JM, Colman H, Soroceanu L, et al: Molecular subclasses of highgrade glioma predict prognosis, delineate a pattern of disease progression, and resemble stages in neurogenesis. Cancer Cell 2006, 9(3):157-173.

26. Pons $\mathrm{S}$, Asano T, Glasheen E, Miralpeix M, Zhang Y, Fisher TL, Myers MG Jr, Sun XJ, White MF: The structure and function of p55PIK reveal a new regulatory subunit for phosphatidylinositol 3-kinase. Mol Cell Biol 1995, 15(8):4453-4465.

27. Xia X, Cheng A, Akinmade D, Hamburger AW: The N-terminal 24 amino acids of the p55 gamma regulatory subunit of phosphoinositide 3-kinase binds Rb and induces cell cycle arrest. Mol Cell Biol 2003, 23(5):1717-1725.

28. Hu J, Liu S, Wang J, Luo X, Gao X, Xia X, Feng Y, Tao D, Wang G, Li X, et al: Overexpression of the $\mathrm{N}$-terminal end of the $\mathrm{p} 55 \mathrm{gamma}$ regulatory subunit of phosphatidylinositol 3-kinase blocks cell cycle progression in gastric carcinoma cells. Int J Oncol 2005, 26(5):1321-1327.

29. Hu J, Xia X, Cheng A, Wang G, Luo X, Reed MF, Fojo T, Oetting A, Gong J, Yen PM: A peptide inhibitor derived from p55PIK phosphatidylinositol 3-kinase regulatory subunit: a novel cancer therapy. Mol Cancer Ther 2008, 7(12):3719-3728.

doi:10.1186/1755-8794-5-34

Cite this article as: Zhou et al:: Genetic and bioinformatic analyses of the expression and function of PI3K regulatory subunit PIK3R3 in an Asian patient gastric

cancer library. BMC Medical Genomics 2012 5:34.

\section{Submit your next manuscript to BioMed Central and take full advantage of:}

- Convenient online submission

- Thorough peer review

- No space constraints or color figure charges

- Immediate publication on acceptance

- Inclusion in PubMed, CAS, Scopus and Google Scholar

- Research which is freely available for redistribution 